\title{
Pickup Probability of Sediment under Log-Normal Velocity Distribution
}

\author{
Fu-Chun $\mathrm{Wu}^{1}$ and Yao-Cheng $\mathrm{Lin}^{2}$
}

\begin{abstract}
This work presents the formulation of the pickup probability for sediment entrainment under the log-normally distributed instantaneous velocity. Herein two mathematical approaches, namely the analytical method and the first-order approximation method, are employed in the theoretical derivation. The results are compared with the published experimental data and the previous pickup probability derived for the normal velocity distribution. The outcome appears to indicate that the pickup probabilities resulting from the two mathematical methods are of the same order of accuracy if the optimal lift coefficients are used. The error analysis implies that the results for the log-normal distribution reveal a significant improvement of accuracy over that for the normal distribution. The overall improvement exceeds $50 \%$.
\end{abstract}

DOI: $10.1061 /($ ASCE)0733-9429(2002)128:4(438)

CE Database keywords: Entrainment; Velocity distribution; Sediment; Error analysis; Probability.

\section{Introduction}

Stochastic methods have long been applied to modeling the hydraulics of open-channel flow and sediment transport (Papanicolaou 1999b). For the past several decades, despite the improved knowledge gained in the area of stochastic hydraulics, there still remains much space for advancement in practical modeling. For instance, the initial entrainment and motion of sediment particles is generally believed to constitute a stochastic process. The instantaneous shear stress, drag, and lift forces induced by the temporal fluctuations of turbulent flow appear to be the main contributors to the stochastic nature of the sediment entrainment problem. Incorporating this concept into his analysis, Einstein (1950) used the pickup probability to derive the bed load function. He defined the pickup probability as the probability of the dynamic lift on a sediment particle being greater than its submerged weight. When such a state is reached, there is a probability that a sediment particle resting on the bed will start to move in the lifting mode. Hence Einstein's pickup probability essentially represents the lifting probability of a sediment particle. In fact, depending on the characteristics of a near-bed flow, the incipient motion of a sediment particle can occur in either one of the following modes, namely, rolling, sliding, or lifting (Cheng and Chiew 1999; Papanicolaou 1999a). Several works have been di-

\footnotetext{
${ }^{1}$ Associate Professor, Dept. of Bioenvironmental Systems Engineering and Hydrotech Research Institute, National Taiwan Univ., Taipei, Taiwan, R.O.C.

${ }^{2}$ Graduate Research Assistant, Dept. of Bioenvironmental Systems Engineering, National Taiwan Univ., Taipei, Taiwan, R.O.C.

Note. Discussion open until September 1, 2002. Separate discussions must be submitted for individual papers. To extend the closing date by one month, a written request must be filed with the ASCE Managing Editor. The manuscript for this technical note was submitted for review and possible publication on November 29, 2000; approved on August 23, 2001. This technical note is part of the Journal of Hydraulic Engineering, Vol. 128, No. 4, April 1, 2002. CASCE, ISSN 0733-9429/2002/4$438-442 / \$ 8.00+\$ .50$ per page.
}

rected toward the calculation of pickup probability. Sun et al. (1997) considered the probability distributions of the moments caused by the drag, lift, and submerged weight to develop a probability equation for particle rolling. Cheng and Chiew (1998) presented a theoretical formulation of the lifting probability.

Following Einstein's definition, Cheng and Chiew (1998) expressed the pickup probability for sediment entrainment in a hydraulically rough flow as the following:

$$
P=P\left(F_{L}>W\right)=P\left(u_{b}^{2}>B^{2}\right)=P\left(u_{b}>B\right)+P\left(u_{b}<-B\right)
$$

where $F_{L}=$ instantaneous lift force acting on a particle= $C_{L}\left(\pi d^{2} / 4\right)\left(\rho u_{b}^{2} / 2\right)$, in which $C_{L}=$ lift coefficient (typically between 0.1 and 0.4$), u_{b}=$ instantaneous velocity approaching the particle on the bed, $d=$ particle diameter, and $\rho=$ density of fluid; $W=$ submerged weight of the particle $=\left(\rho_{s}-\rho\right) g\left(\pi d^{3} / 6\right)$, in which $\rho_{s}=$ density of sediment particle and $g=$ gravitational acceleration; $B=\sqrt{4 \Delta g d / 3 C_{L}}$, in which $\Delta=\left(\rho_{s}-\rho\right) / \rho$. For a fully turbulent flow, the lift coefficient $C_{L}$ is a function of particle shape (Benedict and Christensen 1972) but is independent of the Reynolds number (Coleman 1967). By assuming that the probability density function (pdf) of $u_{b}$ obeys the normal distribution and employing the experimental mean value $\bar{u}_{b}=5.52 u_{*}$ (where $u_{*}=$ shear velocity) and the standard deviation (or turbulence intensity) $\sigma_{u}=2.0 u_{*}$, Cheng and Chiew (1998) obtained the following expression for pickup probability:

$$
\begin{aligned}
P= & 1-0.5 \frac{0.21-\sqrt{\theta C_{L}}}{\left|0.21-\sqrt{\theta C_{L}}\right|} \sqrt{1-\exp \left[-\left(\frac{0.46}{\sqrt{\theta C_{L}}}-2.2\right)^{2}\right]} \\
& -0.5 \sqrt{1-\exp \left[-\left(\frac{0.46}{\sqrt{\theta C_{L}}}+2.2\right)^{2}\right]}
\end{aligned}
$$

where $\theta=$ dimensionless shear stress $=u_{*}^{2} /(\Delta g d)$. Eq. (2) indicates that the pickup probability is only dependent on $\theta$ if $C_{L}$ is known. Cheng and Chiew (1998) selected a value of 0.25 for $C_{L}$ to best fit the reported experimental data. They further compared Eq. (2) with the theoretical formula by Einstein (1950) and the 
empirical equation by Fredsoe and Deigaard (1992). The results reveal that Einstein's formula consistently overpredicts the pickup probability, and Fredsoe-Deigaard's equation gives meaningful results only for $\theta>0.045$ and underestimates the pickup probability for $\theta>0.1$, whereas Eq. (2) tends to underestimate and overestimate the pickup probability for $\theta<0.07$ and $\theta>0.07$, respectively.

The discrepancies between the measured data and Eq. (2) may be attributed to the differences in grain geometry (Naden 1987), sediment availability (Church 1978), and bed roughness (Papanicolaou et al. 2001), among several others. However, we tend to believe that the Gaussian assumption on $u_{b}$ is a main factor because most of the experiments used for verification were conducted under the conditions similar to those for which Eq. (2) is derived. Moreover, previous investigators (Christensen 1965; Yalin 1977) have pointed out that the component of velocity fluctuation in rough turbulent flows follows the normal distribution with a zero mean yet the streamwise instantaneous velocity is more likely to be characterized by the log-normal distribution (Lopez and Garcia 2001). In fact, the log-normal distribution is physically reasonable for the instantaneous velocity because the approaching velocity in the longitudinal direction should be of positive magnitude, which is evidenced by Nelson et al. (1995). The objective of this study is to revise Eq. (2) with the lognormally distributed instantaneous velocity. To this end we employ two mathematical approaches to formulate the new pickup probability. An error analysis is also conducted to show the improved accuracy of the results obtained in the present study.

\section{Revised Pickup Probability}

As the instantaneous velocity $u_{b}$ obeys the log-normal law for 0 $<u_{b}<\infty$, the logarithm of $u_{b}$ is normally distributed as shown in Fig. 1. If $v_{b}$ denotes the logarithm of $u_{b}$ (i.e., $v_{b}=\ln u_{b}$ ), the pdf of $v_{b}$ can be expressed by

$$
f_{v}\left(v_{b}\right)=\frac{1}{\sqrt{2 \pi} \sigma_{v}} \exp \left[-\frac{\left(v_{b}-\bar{v}_{b}\right)^{2}}{2 \sigma_{v}^{2}}\right]
$$

where $\bar{v}_{b}$ and $\sigma_{v}=$ the mean and standard deviation of $v_{b}$, respectively. The pickup probability given in Eq. (1) should be modified as the following:

$$
P=P\left(u_{b}>B\right)=P\left(v_{b}>B^{\prime}\right)=1-P\left(-\infty<v_{b}<B^{\prime}\right)
$$

in which $B^{\prime}=\ln B$. According to the procedures presented in Cheng and Chiew (1998), one can rewrite Eq. (4) as

$$
\begin{aligned}
P & =1-\left[\int_{-\infty}^{\bar{v}_{b}} f_{v}\left(v_{b}\right) d v_{b}+\int_{\bar{v}_{b}}^{B^{\prime}} f_{v}\left(v_{b}\right) d v_{b}\right] \\
& =0.5-0.5 \frac{B^{\prime}-\bar{v}_{b}}{\left|B^{\prime}-\bar{v}_{b}\right|} \sqrt{1-\exp \left[-\frac{2\left(B^{\prime}-\bar{v}_{b}\right)^{2}}{\pi \sigma_{v}^{2}}\right]}
\end{aligned}
$$

To use Eq. (5) for calculating the pickup probability, one needs the mean and the standard deviation in the normal domain (i.e., $\bar{v}_{b}$ and $\sigma_{v}$, respectively). However, the information that the time average value $\bar{u}_{b}=5.52 u_{*}$ and the turbulence intensity $\sigma_{u}$ $=2.0 u_{*}$ is valid for the original (i.e., log-normal) domain. Thus the task to be undertaken is to transform the given values of $\bar{u}_{b}$ and $\sigma_{u}$ into $\bar{v}_{b}$ and $\sigma_{v}$. Herein two methods are used for the transformation, namely, the analytical method and the first-order approximation method. They are described below.

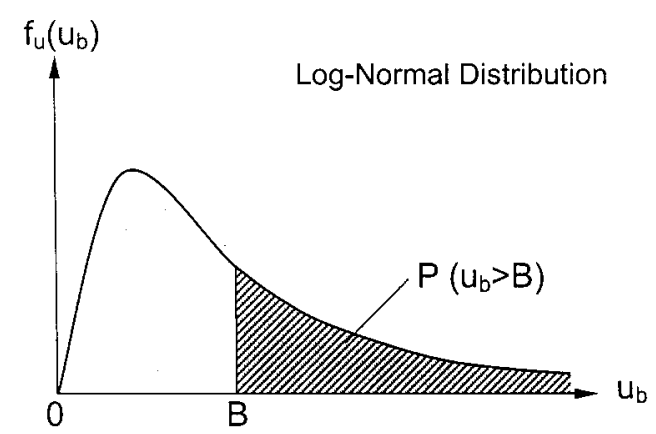

$v_{b}=\ln u_{b}$

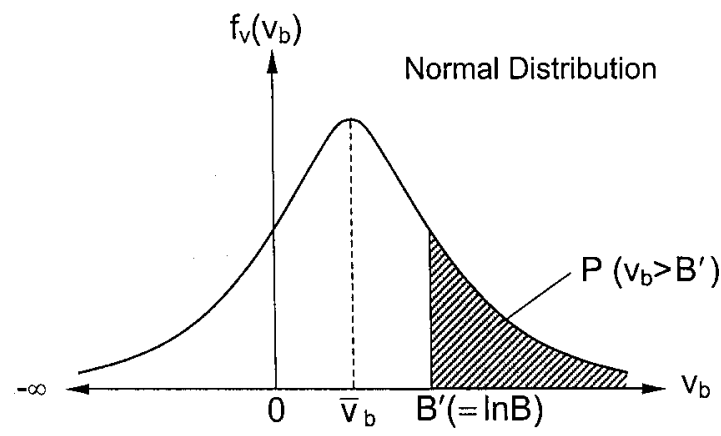

Fig. 1. Schematic diagram showing the transformation of pickup probability between log-normal and normal distributions

\section{Analytical Method}

If $X$ is a random variable whose logarithm $Y$ is normally distributed, it can be shown that both the mean value and the variance of $Y$ vary as a function of the mean and the variance of $X$ (see Appendix I), i.e.,

$$
\begin{gathered}
E[Y]=\ln \left(E[X] / \sqrt{1+V[X] / E^{2}[X]}\right) \\
V[Y]=\ln \left(1+V[X] / E^{2}[X]\right)
\end{gathered}
$$

where $Y=\ln X ; E[X]$ and $V[X]$, and $E[Y]$ and $V[Y]$ are the mean value and the variance of $X$ and $Y$, respectively. Replacing $E[X]$ and $V[X]$ in Eqs. $(6 a)$ and $(6 b)$ with the values of $\bar{u}_{b}$ and $\sigma_{u}^{2}$ yields $\bar{v}_{b}=\ln \left(5.19 u_{*}\right)$ and $\sigma_{v}^{2}=0.123$. Substitution of $\bar{v}_{b}$ and $\sigma_{v}^{2}$ into Eq. (5) results in a revised form of the pickup probability

$$
P=0.5-0.5 \frac{\ln \left(0.049 / \theta C_{L}\right)}{\left|\ln \left(0.049 / \theta C_{L}\right)\right|} \sqrt{1-\exp \left\{-\frac{2}{\pi}\left[\frac{\ln \left(0.049 / \theta C_{L}\right)}{0.702}\right]^{2}\right.}
$$

\section{First-Order Approximation Method}

Let $X$ be a random variable whose mean value $E[X]=\xi$ and $Y$ be a mathematical function of $X$, i.e., $Y=\phi(X)$. With the first-order Taylor series expansion, it has been shown that the mean and the variance of $Y$ can be approximated by the following expressions (see Appendix II):

$$
\begin{gathered}
E[Y]=\phi(\xi) \\
V[Y]=\left[\phi^{\prime}(\xi)\right]^{2} \cdot V[X]
\end{gathered}
$$




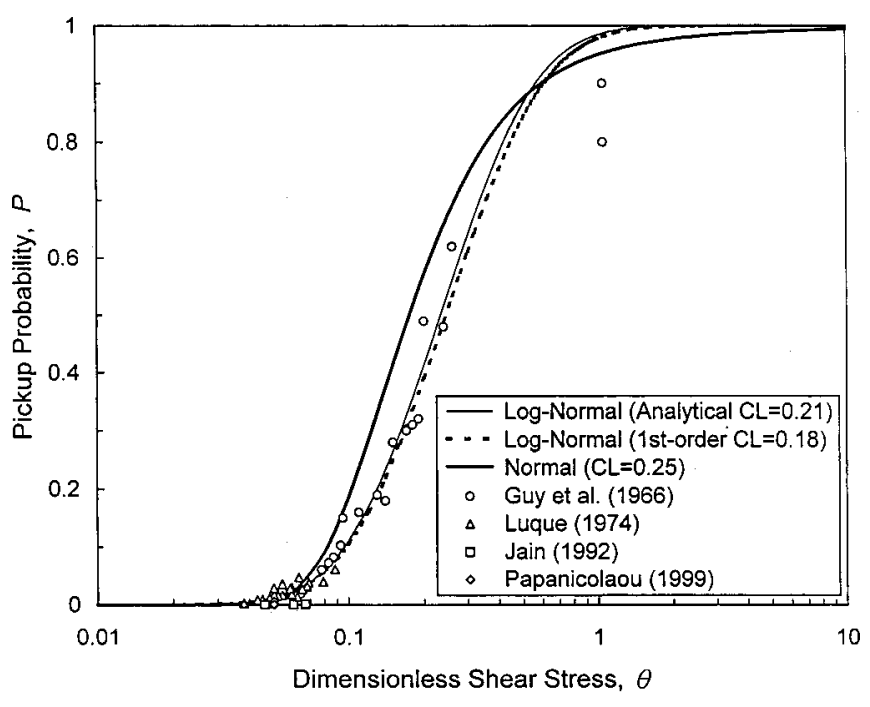

Fig. 2. Comparison of theoretical pickup probabilities with experimental data. The optimal $C_{L}$ values for log-normal (analytical and first-order approximation methods) and normal distributions are 0.21 , 0.18 , and 0.25 , respectively.

Setting $\phi(X)=\ln X$ and replacing $\xi$ and $V[X]$ in Eqs. (8a) and $(8 b)$ with the values of $\bar{u}_{b}$ and $\sigma_{u}^{2}$ gives $\bar{v}_{b}=\ln \left(5.52 u_{*}\right)$ and $\sigma_{v}^{2}$ $=0.131$. Substituting $\bar{v}_{b}$ and $\sigma_{v}^{2}$ into Eq. (5) leads to the following formula for pickup probability

$P=0.5-0.5 \frac{\ln \left(0.044 / \theta C_{L}\right)}{\left|\ln \left(0.044 / \theta C_{L}\right)\right|} \sqrt{1-\exp \left\{-\frac{2}{\pi}\left[\frac{\ln \left(0.044 / \theta C_{L}\right)}{0.724}\right]^{2}\right\}}$

Eqs. (7) and (9) both indicate that, for a given lift coefficient $C_{L}$, the pickup probability is only dependent on the dimensionless shear stress $\theta$.

\section{Results and Discussion}

For comparison, the results of Eqs. (2), (7), and (9) as well as the published experimental data (Guy et al. 1966; Luque 1974; Jain 1992; Papanicolaou 1999a) are demonstrated in Fig. 2. Given that the $C_{L}$ value used by Cheng and Chiew (1998) was based on curve fitting, herein we select appropriate $C_{L}$ values to best fit the experimental data. The optimal $C_{L}$ values for Eqs. (7) and (9) are 0.21 and 0.18 , respectively. The former is close to the $C_{L}$ value selected by Cheng and Chiew (1998) (i.e., 0.25) and the latter is very close to the constant value (i.e., 0.178) used by Einstein and El-Samni (1949) for the flow velocity measured at $0.35 d$ above the theoretical bed level. Later we will also show that the lognormal velocity distribution performs better than the normal distribution even if the same $C_{L}$ value $(=0.25)$ is used for Eqs. (2), (7), and (9). In Fig. 2, one can see that the results of Eqs. (7) and
(9) are almost identical and in better agreement with the experimental data than Eq. (2). To quantitatively investigate the improved accuracy, the Euclidean norm is used as a measure to represent the overall error between the theoretical and experimental results (Conte and de Boor 1980). The Euclidean norm is defined as

$$
\|e\|_{2}=\sqrt{\sum_{i=1}^{n} e_{i}^{2}}
$$

where $e_{i}=$ the difference between the $i$ th data and the theoretical value, and $n=$ the total number of the experimental data. The magnitudes of the Euclidean norm and the coefficient of determination $R^{2}$ for Eqs. (2), (7), and (9) are listed in Table 1, where one may notice that the values of $\|e\|_{2}$ and $R^{2}$ for Eqs. (7) and (9) are identical (i.e., 0.266 and 0.966 , respectively) when the optimal $C_{L}$ values are used. This appears to imply that the pickup probabilities resulting from the analytical and the first-order approximation methods are of the same order of accuracy in case the appropriate lift coefficients can be adopted. The $\|e\|_{2}$ value for Eq. (2) reaches 0.541 , which is much greater than that value for Eqs. (7) and (9) (i.e., 0.266). The $R^{2}$ value for Eq. (2) (i.e., 0.858) is substantially lower than that value for Eqs. (7) and (9) (i.e., 0.966). In summary, the results for the log-normal velocity distribution reveal a significant improvement of accuracy over that for the normal distribution. The outcome of the current study indicates that the overall percentage of improvement is at the level of $51 \%$. On the other hand, when the same $C_{L}$ value (i.e., 0.25) is used, the corresponding $\|e\|_{2}$ values for Eqs. (7) and (9) are 0.432 and 0.794, while the corresponding $R^{2}$ values are 0.910 and 0.807 . The performance of the log-normal distribution is still superior to that of the normal distribution, although the first-order approximation is not as good as the analytical method. The claim made here is based on the comparison with the experimental data. Currently the available data are limited, especially for the range of high shear stress. Thus more data are needed for validation before a general conclusion can be drawn.

\section{Conclusions}

In this study we revised the pickup probability of sediment entrainment with the log-normal velocity distribution. Two mathematical approaches (i.e., the analytical and the first-order approximation methods) were used for the formulation of the pickup probability. The modified results were compared with the experimental data and the previous pickup probability derived for the normal velocity distribution. It is shown that the accuracy of the results gained from the analytical and the first-order approximation methods is nearly identical in case the optimal lift coefficients are used. The error analysis also indicates that the modified results for the log-normal distribution reveal a significant improvement over that for the normal distribution. The overall improvement of the accuracy exceeds $50 \%$. In the present study the

Table 1. Euclidean Norm and Coefficient of Determination for Different Probability Distributions

\begin{tabular}{|c|c|c|c|c|c|}
\hline \multirow[b]{3}{*}{ Probability distribution } & \multicolumn{4}{|c|}{ Log-normal } & \multirow{3}{*}{$\begin{array}{c}\text { Normal [Eq. (2)] } \\
C_{L}=0.25\end{array}$} \\
\hline & \multicolumn{2}{|c|}{ Analytical [Eq. (7)] } & \multicolumn{2}{|c|}{ First-order Approximation [Eq. (9)] } & \\
\hline & $C_{L}=0.21$ & $C_{L}=0.25$ & $C_{L}=0.18$ & $C_{L}=0.25$ & \\
\hline$\|e\|_{2}$ & 0.266 & 0.432 & 0.266 & 0.794 & 0.541 \\
\hline$R^{2}$ & 0.966 & 0.910 & 0.966 & 0.807 & 0.858 \\
\hline
\end{tabular}


optimal choice of the $C_{L}$ value for sediment entrainment is based on the best fitting to the experimental data. However, the dependence of the $C_{L}$ value on the flow condition and grain geometry (such as particle shape and grading of nonuniform sediment) is of great importance and remains to be investigated in a future study.

\section{Appendix I. Derivation of Eq. (6)}

Given that $E[Y]=\eta$ and $V[Y]=\sigma^{2}$, the pdf of $Y$ can be expressed as

$$
f_{Y}(y)=\frac{1}{\sqrt{2 \pi} \sigma} \exp \left[-\frac{(y-\eta)^{2}}{2 \sigma^{2}}\right] \text { for }-\infty<y<\infty
$$

Since $Y=\phi(X)=\ln X$, one can obtain the pdf of $X$ by the chain rule and Eq. (11), i.e.,

$$
\begin{aligned}
f_{X}(x) & =f_{Y}[\phi(x)] \frac{d[\phi(x)]}{d x} \\
& =\left\{\frac{1}{\sqrt{2 \pi} \sigma} \exp \left[-\frac{(\ln x-\eta)^{2}}{2 \sigma^{2}}\right]\right\} \frac{1}{x}
\end{aligned}
$$

$$
\text { for } 0<x<\infty
$$

Using Eq. (12) and defining that $\lambda=e^{\eta}$ (i.e., $\eta=\ln \lambda$ ), one can express the mean value of $X$ as the following:

$$
E[X]=\int_{0}^{\infty} x \cdot f_{X}(x) d x=\int_{0}^{\infty} \frac{1}{\sqrt{2 \pi} \sigma} \exp \left[-\frac{\left(\ln \frac{x}{\lambda}\right)^{2}}{2 \sigma^{2}}\right] d x
$$

Changing the variable to replace $\ln (x / \lambda)$ with $\tau$, one can modify Eq. (13) to gain the mean value of $X$, i.e.,

$$
\begin{aligned}
E[X] & =\lambda \exp \left(\sigma^{2} / 2\right) \int_{-\infty}^{\infty} \frac{1}{\sqrt{2 \pi} \sigma} \exp \left[-\frac{\left(\tau-\sigma^{2}\right)^{2}}{2 \sigma^{2}}\right] d \tau \\
& =\exp \left(\eta+\sigma^{2} / 2\right)
\end{aligned}
$$

With the same approach, one can further show that $E\left[X^{2}\right]$ $=\exp \left(2 \eta+2 \sigma^{2}\right)$. From the relationship $V[X]=E\left[X^{2}\right]-E^{2}[X]$, the following result can be obtained

$$
V[X]=\left[\exp \left(2 \eta+\sigma^{2}\right)\right] \cdot\left[\exp \left(\sigma^{2}\right)-1\right]
$$

The combinations of Eqs. (14) and (15) would lead to the expressions given in Eq. (6).

\section{Appendix II. Derivation of Eq. (8)}

Given $Y=\phi(X)$, the Taylor series expansion of $Y$ about $\xi$ can be written as

$$
Y=\phi(\xi)+\phi^{\prime}(\xi) \cdot(X-\xi)+\phi^{\prime \prime}(\xi) \cdot(X-\xi)^{2} / 2+\cdots
$$

The first-order approximation of Eq. (16) is

$$
Y=\phi(\xi)+\phi^{\prime}(\xi) \cdot(X-\xi)
$$

Since $E[X]=\xi$, taking the expected value of Eq. (17) leads to Eq. $(8 a)$, i.e.,

$$
E[Y]=\phi(\xi)+\phi^{\prime}(\xi) \cdot E[X-\xi]=\phi(\xi)
$$

The difference in Eqs. (17) and (18) becomes

$$
Y-E[Y]=\phi^{\prime}(\xi) \cdot(X-\xi)
$$

According to the definition of variance, the square of Eq. (19) can be transformed into Eq. ( $8 b)$, i.e.,

$$
\begin{aligned}
V[Y] & =E\left[(Y-E[Y])^{2}\right]=\left[\phi^{\prime}(\xi)\right]^{2} \cdot E\left[(X-\xi)^{2}\right] \\
& =\left[\phi^{\prime}(\xi)\right]^{2} \cdot V[X]
\end{aligned}
$$

\section{Notation}

The following symbols are used in this paper:

$$
\begin{aligned}
B & =\sqrt{4 \Delta g d / 3 C_{L}} \\
B^{\prime} & =\ln B \\
C_{L} & =\text { Lift coefficient; } \\
d & =\text { diameter of a particle; }
\end{aligned}
$$

$E[X]=$ expected value of the random variable $X$;

$\|e\|_{2}=$ Euclidean norm of the error;

$F_{L}=$ instantaneous lift force;

$f_{v}\left(v_{b}\right)=$ probability density function (pdf) of $v_{b}$;

$g=$ gravitational acceleration;

$P=$ pickup probability of sediment;

$R^{2}=$ coefficient of determination;

$u_{b}=$ instantaneous velocity approaching a bed particle;

$\bar{u}_{b}=$ time-mean value of $u_{b}$;

$u_{*}=$ shear velocity;

$V[X]=$ variance of the random variable $X$;

$v_{b}=\ln u_{b}$

$\bar{v}_{b}=$ mean value of $v_{b}$;

$W=$ submerged weight of a sediment particle;

$\Delta=\left(\rho_{s}-\rho\right) / \rho$

$\theta=$ dimensionless shear stress $=u_{*}^{2} /(\Delta g d)$;

$\rho=$ density of fluid;

$\rho_{s}=$ density of sediment particle;

$\sigma_{u}=$ standard deviation of $u_{b}$ (or turbulence intensity); and

$\sigma_{v}=$ standard deviation of $v_{b}$.

\section{References}

Benedict, B. A., and Christensen, B. A. (1972). "Hydrodynamic lift on a stream bed." Sedimentation, H. W. Shen, ed., Fort Collins, Colo., 5.1-5.17.

Cheng, N.-S., and Chiew, Y.-M. (1998). "Pickup probability for sediment entrainment." J. Hydraul. Eng., 124(2), 232-235.

Cheng, N.-S., and Chiew, Y.-M. (1999). "Closure to discussion of 'Pickup probability for sediment entrainment.' " J. Hydraul. Eng., 125(7), 789-789.

Christensen, B. A. (1965). "Discussion of 'Erosion and deposition of cohesive soils.' " J. Hydraul. Div., Am. Soc. Civ. Eng., 91(HY5), 301308.

Church, M. A. (1978). "Palaeohydrological reconstructions from a Holocene valley fill." Fluvial sedimentology, A. D. Miall, ed., Canadian Society of Petroleum Geologists, Alberta, Canada, 743-772.

Coleman, N. L. (1967). "A theoretical and experimental study of drag and lift forces acting on a sphere resting on hypothetical stream bed." Proc., 12th Congress, IAHR, Fort Collins, Colo., 3, 185-192.

Conte, S. D., and de Boor, C. (1980). Elementary numerical analysis, McGraw-Hill, New York.

Einstein, H. A. (1950). "The bed load function for sediment transportation in open channel flows." Tech. Bull. 1026, U.S.D.A., Washington, D.C. 
Einstein, H. A., and El-Samni, E. A. (1949). "Hydrodynamic forces on a rough wall." Rev. Mod. Phys., 21, 520-524.

Fredsoe, J., and Deigaard, R. (1992). Mechanics of coastal sediment transport, World Scientific, River Edge, N.J.

Guy, H. P., Simons, D. B., and Richardson, E. V. (1966). "Summary of alluvial channel data from flume experiments, 1956-1961." U.S.G.S. Professional Paper, 462-I.

Jain, S. C. (1992). "Note on lag in bedload discharge." J. Hydraul. Eng., 118(6), 904-917.

Lopez, F., and Garcia, M. H. (2001). "Risk of sediment erosion and suspension in turbulent flows." J. Hydraul. Eng., 127(3), 231-235.

Luque, R. F. (1974). Erosion and transport of bed load sediment, Delft University of Technology, Delft, The Netherlands.

Naden, P. (1987). "An erosion criterion for gravel-bed rivers." Earth Surf. Processes Landforms, 12, 83-93.
Nelson, J. M., Shreve, R. L., McLean, S. R., and Drake, T. G. (1995). "Role of near-bed turbulence structure in bed load transport and bed form mechanics." Water Resour. Res., 31(8), 2071-2086.

Papanicolaou, A. N. (1999a). "Discussion of 'Pickup probability for sediment entrainment.' " J. Hydraul. Eng., 125(7), 788-789.

Papanicolaou, A. N. (1999b). "Stochastic considerations in hydraulics." J. Hydraul. Eng., 125(12), 1229-1330.

Papanicolaou, A. N., Diplas, P., Dancey, C. L., and Balakrishnan, M. (2001). "Surface roughness effects in near-bed turbulence: Implications to sediment entrainment." J. Eng. Mech., 127(3), 211-218.

Sun, Z., Xie, J., Duan, W., and Xie, B. (1997). "Incipient motion of individual fractions of nonuniform sediment." J. Hydr. Eng., Chin. Hydr. Eng. Soc., 97(10), 25-32 (in Chinese).

Yalin, M. S. (1977). Mechanics of sediment transport, Pergamon, Tarrytown, N.Y. 